\title{
Review of Concrete Appearance Quality, Defect Prevention and the Treatment Methods
}

\author{
Chunfeng Li, Yubo Yang, Chenglin Yao, Zhongjun Deng, Yongmei Jia, Bin Zhi \\ China Institute of Water Resources and Hydropower Research \\ Beijing, 100048, China \\ Email: 58748098@qq.com
}

\begin{abstract}
Concrete is widely used in hydraulic structure, which may lead to the concrete appearance quality defects. Treatment methods for the appearance quality defects are different due to different forming reason. Combining with engineering practice, this paper points out a large number prevention and control measures of concrete appearance quality defects, and summarizes the reasons, prevention measures and handling methods. The paper also discovers the appearance quality defects in similar engineering and in its process, and tries to improve concrete structures' durability.
\end{abstract}

Keywords: Concrete, Appearance defects, Cause, Prevention and treatment

\section{BACKGROUND OF RESEARCH}

Concrete is a mixture of cementing material, water and coarse aggregate in proper proportion, mixed with a mixture of hydrated and thermally reacted at a certain time, and then solidified and hardened.

Concrete has the strength and durability required by engineering. Since 1820 s, it has been applied to many fields, such as building construction, road and bridge, water conservancy, tunnel, rescue and so on. But because of a series of problems in the concrete construction of complex factors inevitable operational errors in the construction process, and concrete shrinkage, deformation, hardening of the concrete forming prone to various surface defects, if not timely treatment, with the passage of time, not only affects the appearance of the building, and even affect their life.

\section{CAUSE OF APPEARANCE QUALITY DEFECT}

\section{A. Honeycomb}

Mix properly, gravel gradation is not good, resulting in a small mortar stone; uneven stirring, separation of aggregate and mortar; template damage or block lax, or formwork is not strong, resulting in leakage; a concrete thickness is too large, resulting in uneven vibration, not in place; concrete falling from the height of the pouring surface is too high, resulting in stone and cement under the action of gravity separation, resulting in stone to heap; the vibration time is not sufficient, not excluded, bubble collapse through small spacing or too dense steel bars, inadequate protection of reinforced.

\section{B. Pitting Surface}

The template cleaning is not clean, or stripping prematurely, formwork and concrete adhesion; not watering wood template moist, make the concrete surface dehydration powder; the vibration time is not sufficient, the bubble does not exclude or not clean.

\section{Holes}

Concrete segregation, vibrating imperfect; reinforcement spacing is too small, or the aggregate size is too large, resulting in dust and vibration is difficult, and the blowhole; hole, bottom template does not vent or vent plug, internal bag or other impurities.

\section{Tendon Leakage}

Steel skeleton processing allowed, top stick template, concrete pouring can not fill the gap; lack of protective layer pad or pad displacement, the reinforcement and formwork close to the protective layer; or the small concrete slurry leakage, leakage of vibration; aggregate size is too large, the vibration is not sufficient, reinforced by the steel overhead

There is no concrete between the bar and the template.

\section{E. Joint Dislocation}

The template is placed at the wrong time, the adjacent template stiffness is uneven, the strength of the pull rod or support is insufficient, or the individual support and rod loosening cause the template to shift in the pouring, and the concentrated vibration leads to the pull rod or support loosening.

\section{F. Root Rot}

The water cement ratio of concrete will be over the stone bottom side of the mold wall; the root run mode leakage, cause rot; pouring height is too high, focus on a concrete material, concrete segregation or gravel catch pile; horizontal bars close to the side wall template; mold cleaning is not clean, too much water, diluted to concrete. It's easy to run down the sand slurry, causing accumulation caused rot.

\section{G. Sand Line}

Horizontal sand line horizontal seam not close due to template plasma leakage caused by sand; vertical lines are due to concrete collapse through large, and vibration when the two adjacent rod working radius and template not overlap, water along the template to form; concrete gradation unreasonable is also an important reason; mechanism in the large amount of gravel dust. 


\section{H. Crack}

The water-cement ratio is too large, and the surface produces pores and cracks. Too much cement, shrinkage crack; Poor or untimely maintenance, surface dehydration, dry shrinkage cracks; The slump is too high, the pouring is too thick, and the floating surface of the slurry cracks. To pry the concrete with undue force when removing the mould too early; The concrete surface is not compacted; Steel reinforcement protection layer is too thin. The absence of stirrup and temperature bar makes the concrete crack. Large volume concrete has no measures to reduce the temperature difference between inside and outside. There is no reinforcing steel bar at the entrance corner.

\section{Missing Edge}

The design of the template does not take into account the factor of preventing the Angle loss. The wooden mould is not wetted in advance, and the expansion of the wooden mould after pouring causes the concrete Angle to crack. The formwork is not properly sewed and the pulp is leaking. The template is not coated with an isolator or poorly painted, resulting in adhesion of the remover. Too early die removal, improper method and procedure; Poor maintenance.

\section{PREVENTION AND TREATMENT OF APPEARANCE QUALITY DEFECTS}

\section{A. Honeycomb}

\section{1) Prevention and control measures}

To strictly control the reasonable mix, strict measurement, regular inspection of concrete mix stirring time and speed; choose the right; two times shall be arbitrarily mixing water; layered material and thickness of each layer is greater than $30 \mathrm{~cm}$, vibrated, to prevent leakage of vibration; the integrity of the template, pouring in at any time to check for any leakage of the.

\section{2) Treatment measures}

For small cell, wash clean with 1:2 cement mortar compaction, can also be used epoxy resin mortar repair, curing 7 days fineness is consistent; large honeycomb, chisel to weak loose parts, wipe with a steel brush, pressure water washing, the surface clean and moist after repair with high grade concrete; deep cellular on the surface of mortar or concrete closed after cement grouting treatment.

\section{B. Pitting Surface}

\section{1) Prevention and control measures}

The template must be cleaned up, not allowed to be adhered to impurities. The wood formwork should be straight and wet before pouring, the steel template should be evenly coated with better isolating agents, reduce the viscosity of concrete, and the concrete must be layered and evenly vibrated according to the operation rules, so as to prevent [1] from leakage.

\section{2) Treatment measures}

For the larger parts of the repair, that is, after washing water, use cement to smooth.

\section{Holes}

\section{1) Prevention and control measures}

In the reinforcement of dense or complex embedded parts can be used in places where the fine stone concrete, with a small diameter vibrator vibration compacting; have reserved holes and pipes from both sides of material, vibration should be inserted quickly pulled slowly into concrete; prevent sundries, such as tools and templates; strengthen the construction technology and quality management check [1].

\section{2) Treatment measures}

The loose concrete around the hole is chiseled and washed with high pressure water. After wetting, it is carefully poured, tamped and maintained with fine concrete with a high strength grade. The treatment plan shall be formulated by the construction unit and shall be submitted to the supervision, construction unit and the construction quality management department for approval or approval.

\section{Tendon Leakage}

\section{1) Prevention and control measures}

To prevent the protective layer block off or dislocation; don't avoid concrete formwork, missing edge off angle; pouring and vibration to avoid collision with steel.

\section{2) Treatment measures}

The surface leaking tendon after washing, with cement mortar compaction, $1: 2$ or $1: 2.5$ pay attention to maintenance; if the tendon leakage is deep, should be the weak part of the cut concrete and prominent, then rinse, with fine stone concrete filling compaction had high strength grade, and careful maintenance.

\section{E. Joint Dislocation}

\section{1) Prevention and control measures}

Try to use high rigidity materials for construction, such as construction formwork must buy better quality of the template, and the need to control the turnover times; after the installation template carefully check whether the formation of the joint, installation is firm; should not be too large for the tension rod, easy to cause the template deformation, should increase some support, which can ensure the template is straight, no deformation; bottom formwork can be used after the anchor anti pressure jack back pressure, so that the template and concrete joint is more strict, not easy to leak slurry; joint position and template the template can use wood glue or glass glue, the seam is completely sealed, the joints without leakage; pay attention to vibration rod position construction, should be about $5 \mathrm{~cm}$ from the template of vibration; elevation for each segment of strict control, strict alignment of whole bridge monitoring, and the monitoring data in time the adjustment of each segment of the vertical mold elevation.

\section{2) Treatment measures}

The protruding parts are chiseled or polished at the wrong platform, so that the size of the wrong table and the deviation of the cross section are within the range of the standard deviation. When the chiseled parts are washed with water, the surface is leveled with $1: 2$ or 1:2.5 cement mortar. 


\section{F. Root Rot}

\section{1) Prevention and control measures}

Adjust the water cement ratio of concrete, so that all the space of the shell mold filled with concrete, to eliminate concrete water cement ratio is too large to produce root rot; template stitching should be close to pull the bolt to tighten, the sponge plugging, prevent leakage; pick a certain vibration experience, have high responsibility the heart of the skilled workers to operate, including formwork, pouring, vibrating, demoulding and curing.

\section{2) Treatment measures}

If the permeability of root rot is longer, larger, should adopt the pressure grouting reinforcement; if the local produce rot or range is small, which belongs to the superficial (depth less than $2 \mathrm{~cm}$ ) rot, the mortar concrete wall on a higher level than the strength of the artificial hand pressure compacting repair; leakage vibration range is very large, must be cut in addition to all real and concrete, formwork repouring high strength concrete wall; Ministry of water, cause rot, scraping floating sand to compacting parts by mortar compaction ratio of concrete strength grade.

\section{G. Sand Line}

\section{1) Prevention and control measures}

Careful design of concrete mix ratio; timely treatment of gaps between formwork and formwork flange and panel; strictly control the content of stone powder in sand and gravel; use good quality release agent; avoid rainy day construction, master concrete vibration technology and maintain timely.

\section{2) Treatment measures}

Selection of fine sand, and add white cement and cement black in fine sand (amount of white cement and cement for the black sand weight of $70 \%$ and $20 \%$ respectively), add water mixing evenly, and then uniformly mixing the mortar with a spatula onto the sand line, polished with fine gauze, and then with water the black and white cement, cement and sand, wipe it again, and finally covered with plastic film curing.

\section{H. Cracks}

\section{1) Prevention and control measures}

In terms of design, the combination of resistance and release in the design can avoid the stress concentration caused by the sudden change of structural section, adopt the technology of compensating shrinkage concrete, pay attention to the control measures of structural reinforcement and so on.

Material selection is based on the selection of suitable concrete admixture strength grade, cement variety, grade and grade of fine sand and stone materials, and active use of admixture and concrete exterior control measures.

The concrete mixing ratio is designed from dry shrinkage, slump, water consumption, cement dosage, water-cement ratio, sand ratio, and control measures such as air attractant or water reducing agent.
Construction is controlled from the installation and removal of formwork, preparation, transportation, casting and maintenance of concrete.

In terms of management, the scientific crack control standards should be determined and the construction progress should be reasonably selected. In the environmental aspect, attention should be paid to the construction season, the temperature and humidity of the environment, strictly control the site slump, wind prevention, and timely maintain close contact with weather stations.

\section{2) Treatment measures}

The main methods of concrete crack treatment are surface treatment, filling method, grouting method, structural reinforcement method, concrete replacement method, electrochemical protection method and bionic self-healing method.

\section{Missing Edge}

\section{1) Prevention and control measures}

The formwork before pouring concrete should be fully wet or coated with spacer. Stipulate concrete maintenance; When removing the side non-bearing formwork, the concrete should be strong enough. When removing the mold, do not force too hard or too fast, pay attention to protecting the corners; When hoisting, it is forbidden to hit the edge Angle of the template. Strengthen product protection.

\section{2) Treatment measures}

Small missing edge off the Angle, can be the place of loose stone chisel, with steel wire brush clean, clean water and sufficient moisture after washing, with cement mortar wipe to complete. The larger missing edges drop the Angle, after washing and chipping clean, re-support the mold with a high strength grade of fine stone concrete fill compaction, and maintenance.

\section{CONCLUSION}

There are many kinds of defects in concrete appearance, which are unavoidable in the construction. In the construction of a strict quality management system, clear quality objectives, the use of the correct construction technology can reduce appearance quality defects. Once the defect appears, the correct treatment method is found in time to repair it carefully to avoid its expansion and deterioration, so as to ensure the perfect image of the concrete project.

\section{REFERENCE}

[1] Lin Jungui. Analysis on the causes and prevention measures of the surface quality defects of concrete [J]. China's scientific and technological information. 2012 (18).

[2] Duan Pin. A brief discussion on the reasons and control methods of the concrete staggered platform in the cantilever box girder $[\mathrm{J}]$. The frontier of architecture research. 2012 (12).

[3] Chu Lili. Study on the treatment and prevention of root rot of concrete in Hydraulic Engineering [J]. Doors and windows. 2013 (11).

[4] Zhang Jinbao. Causes and prevention of sand line formation on concrete surface of bridge and culvert [J]. Qinghai science and technology. 2011 (02). 
[5] Han Xinhua. Construction method of repairing and strengthening the defect of concrete surface quality $[\mathrm{J}]$. Shanxi architecture. 2005 (13).

[6] Song Taiyu, Hao Gaiyun. Analysis and treatment of surface quality defects of concrete $[\mathrm{J}]$. Water conservancy construction and management. 2016 (6) 